Editorial paper

\title{
Plasma-inspired biomaterials
}

\author{
Uroš Cvelbar $^{1}$, Cristina Canal$^{2}$, Masaru Hori ${ }^{3}$ \\ ${ }^{1}$ Jozef Stefan Institute, Jamova cesta 39, SI-1000, Ljubljana, Slovenia \\ ${ }^{2}$ Biomaterials, Biomechanics and Tissue Engineering, Materials Science and Metallurgy Dpt., Technical \\ University of Catalonia (UPC), Avda. Diagonal 647, 08028 Barcelona, Spain \\ ${ }^{3}$ University of Nagoya, Nagoya, Japan
}

The first question which arises when one looks at the title of this special issue is "What are plasmainspired biomaterials” and what is this special issue of Journal of Physics D: Applied Physics (JPhysD) all about? Peculiar as it may seem, from a philosophical point of view inspiration is something that influences or connects two at first glance opposite things - plasma and biomaterials. On the one hand, plasma as discharge state of the gas is considered nowadays as a cutting edge tool which can manipulate objects at the atomic or molecular scale. to functionalize, modify, synthesize, embedded, etc. (Figure 1). On the other hand, biomaterials are substances that have been engineered to possess certain properties which can control the interactions with components of living systems, inducing favourable response from the biological entities, and as such can direct the course of a therapy or diagnostic procedure [Williams, 2009]. In this respect, plasmas can be used to initiating even more favourable or selective responses, making the biomaterials even more suitable for their interaction biological entities.

The most appropriate plasmas for initiation of these responses and modifications are so-called cold plasmas, which are generated either at reduced or atmospheric pressures. Cold plasmas have been employed to tailor the surfaces of materials for decades. With the increasing relevance of biomaterials, which can augment or replace partially or totally any tissue, organ or function of the body, in order to maintain or improve the quality of life of the individuals, the potential of plasmas in the field is extraordinary and has seen some significant advances in last years.

This special issue on Plasma-inspired biomaterials wishes to highlight the most recent developments in the area where plasmas are used directly or indirectly for preparation of biomaterials or biocompatible surfaces. The issue covers all aspects of research which connect plasma and biomaterials, ranging from plasma preparation of biomaterials for different applications (hard tissues such as bone, and soft tissues), including drug delivery applications, antibacterial coatings, and focuses also on biological interactions of the novel plasma-prepared surfaces with bacteria, cells and tissues. Novel developments for diagnostics 
and sensing are also included in this issue. All these contributions to the issue are briefly summarized in the four coming sections.

\section{Novel biomaterials, implants and grafts}

Bone is one of the most transplanted tissues, and as such, bone grafts and bone implants raise significant attention. Titanium is often the material of choice for bone implants given its excellent biocompatibility. An issue not so often taken into account is that temporary implants have to fulfil different requirements as permanent implants. For instance, the former should avoid ossteointegration, as they need to act as support materials only until bone has healed. As such, different plasma processes were investigated to produce fluorocarbon polymer films which were able to prevent osteoblast adhesion in vitro, with comparable in vivo inflammatory response with uncoated materials [Finke]. In fact, the surface of Ti is oxidized, so that in fact, a thin layer of $\mathrm{TiO}_{2}$ is exposed to the biological media. To ascertain its potential to enhance osteoblast-like cell response, oxygen plasmas were studied on $\mathrm{TiO}_{2}$ with promising results following cleaning and functionalisation of the surface.[Junkar] Another way to enhance ossteointegration is by precipitation of calcium phosphates onto titanium surfaces. To enhance the efficiency of the process evaluated by Mahanti et al., it has been shown that the parameters should be adjusted to induce surface heating and plasma-mediated surface reactions. [Mahanti]

From bone implants to bone grafts, resorbable calcium phosphate bone grafts have excellent features but controlling drug delivery from such matrices is a complicated issue. To that aim, low pressure plasma processes have been investigated with biocompatible plasma polymers, successfully reducing antibiotic release rates [Labay]. A similar approach investigated elution of another antibiotic from polymeric substrates by multilayer siloxane coatings generated by plasma which extended the drug release duration after coating [Dowling]. In both cases the antimicrobial activity of the drug was conserved after the plasma treatments investigated. To this regard, in views of antibiotic sensitivity tests, atmospheric plasma jet was employed to modify cellulose. [Pawlat]

Cold plasmas can be an excellent alternative to the still widely used wet chemistry to conjugate biomolecules onto biomaterials. For instance, electrospun PLLA fibers were plasma-functionalized prior to antibody immobilisation with great success. [Dolci] But plasma itself can be employed beyond functionalisation, and be used to build the scaffolds, including nanostructured surfaces like vertically interconnected carbon nanowalls with good biocompatibility with bone forming cells. [Ion] Orthesis are also of interest in this area, and as such, the increased adhesion of Polydimethylsiloxane for medical use achieved by atmospheric pressure plasma was significant though with the limitation of time-decay that could be controlled with the application of a further adhesive layer. [Jofre-Reche] 


\section{Tailoring surface-cell response}

Tailoring cell response to surfaces is one of the most difficult tasks in surfaces engineering, since there are many parameters which can influence how cells will behave. In previous years, researchers have already found clues that functional groups, surface temperatures, surface charge, material properties and composition, stiffness, topography, etc. can influence the response of interacting biological cells. A relatively straightforward way to tailor cell response is by playing with the topography of biocompatible materials. Herein, plasmas present an inexhaustible source of options to design nanostructured surfaces and tailor their response. This can be achieved for various biological entities like proteins and enzymes as well as living cells and tissues. [Levchenko] Similarly, one can also mimic nature with nanostructured films that have been investigated by combining the gas aggregation of nanoparticles and coating overdeposition. [Shelemin] By adjusting plasma parameters the surface morphology can change as a function of roughness height, correlation length and fractal dimensions on polymer surfaces. Such surfaces can differently influence cell attachment and proliferation. [Bourkoula] However, plasma-surface interactions can result also in change of surface-electric properties. Namely, electrical conductivity and surface energy were identified to play a key role in different time scales of the cell proliferation processes. [Amjed Javid]

\section{Antimicrobial surfaces}

On the contrary to the expected favourable eucariotic cell responses of plasma treated surfaces, where proliferation, cell growth, attachment, etc. are desired, the focus in antimicrobial surfaces is repulsion and/or prokaryotic cell death. Plasma generated antimicrobial coatings include nanocomposite films, plasma engineered drug delivery coatings, etc. Compared to traditional wet chemistry, plasma deposition and polymerization are new promising approaches for synthesis of nanocomposite films with the advantage of dry phase processes, effective use of chemical and wide applicability, as reviewed by Nikiforov et al. [Nikiforov] Alternatively to highly resistive antimicrobial coatings, enhanced effects of nanoparticles which account for antibacterial properties of textiles can be achieved also by plasmaenhanced binding of nanoparticles. [Primc] More complex systems can be further tailored by plasmas in various steps of the process. Therein, to obtain antimicrobial surfaces, plasma can be used first to synthesize and then functionalize nanoparticles as well as attaching biomolecules such as antibodies more efficiently. [Viswan] Instead, plasma etching is another way of designing complex structures, such as nanoscale surfaces like silicon nanograss with tuned hydrophilicity to regulate the antimicrobial activity. [Ostrikov] However, one needs to look beyond pure bactericidal effects of surfaces, and antifungal 
activity is also a requirement for many applications. Plasma polymer coatings derived from chlorinated hydrocarbon molecules may therefore offer another promising solution to prevent yeast or mould biofilm formation. [Lamont-Friedrich]

\section{Generation and detection of reactive oxygen and nitrogen species (RONS)}

At the end of the line, to tailor the biological response one has first to understand the underlying mechanisms and species created in the applied plasmas. Due to the lack of proper detection tools, this can be a pretty tricky task. This tools of detection vary for low or atmospheric pressure plasmas as well as depend on the gas or liquid phase. A well known technique used for gas phase diagnostics is optical emission spectroscopy, which can be used to probe any plasmas as well as help in understanding the mechanisms of plasma interaction with biological materials. This has been employed in this special issue to evaluate differences in blood coagulation behaviour during different plasma treatments. [Yamada] Increasing complexity and measurements occur when one measures gas-liquid or only liquid phase in systems with biological materials. To try overcoming this complexity models are required, such as that in the work of Oh et al., where the interactions of plasmas with biological tissues are simulated thorough an agarose target, reflecting the choice of process gas that can have a profound effect on concentration of RONS. The effects on cells are even more complex. It is known that plasma induces cell membrane permeabilization [Sasaki] and liposomes have been successfully evaluated in a very interesting approach as membrane models for cell inactivation [Maheux].

The path of cold plasmas for design and tailoring of biomaterials has only started, and there is a long way to the top of summit with exciting new horizons of research ahead. Furthermore, the bench models and lab experiments will have to be transferred into biotechnological and medical practice. The editors of this issue hope that this issue will inspire the readers to undertake more research in this field to unravel yet unsolved challenges. 


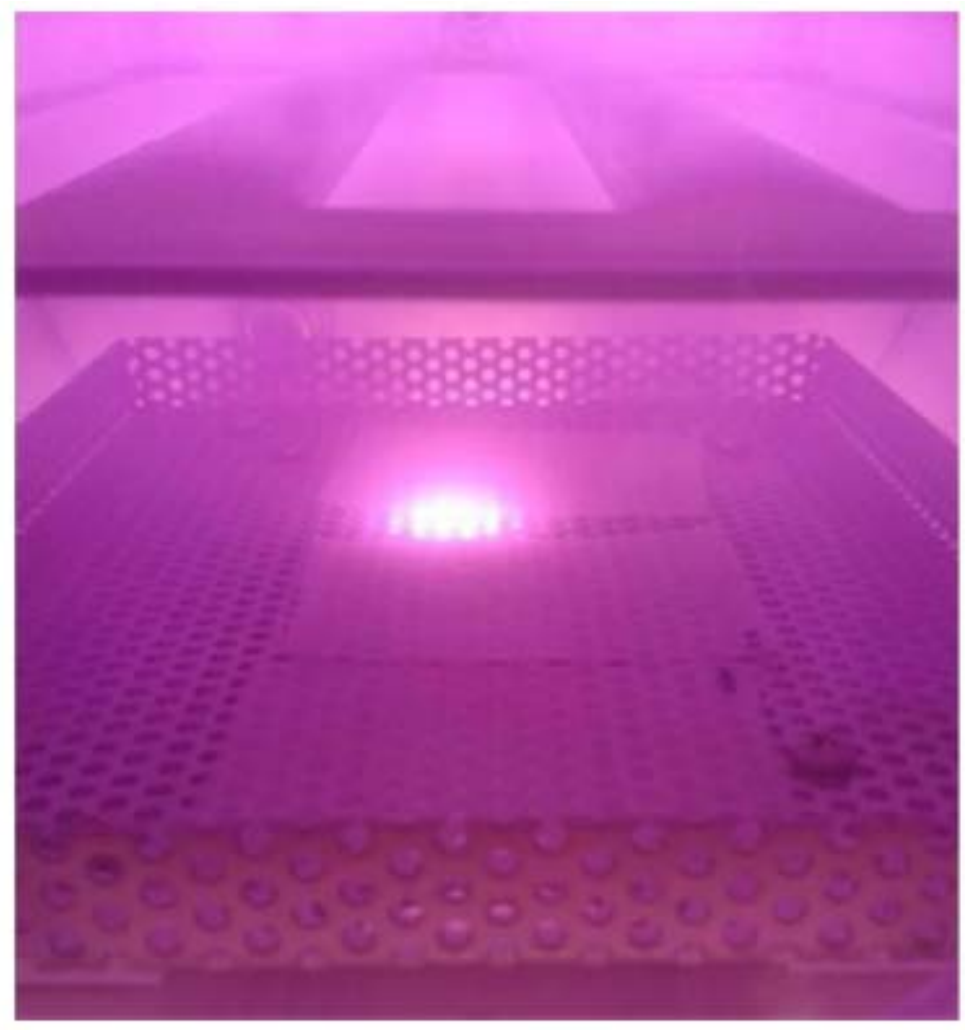

Figure 1. Low pressure RF plasma system for modification of biomedical polymers.

\section{References}

[1] Williams D 2003 Revisiting the definition of biocompatibility Med. Device Technol. 14 10-3 [2] Finke B et al 2016 Plasma-deposited fluorocarbon polymer films on titanium for preventing cell adhesion: as surface finishing for temporary used orthopaedic implants J. Phys. D: Appl. Phys. 49234002

[3] Junkar I et al 2016 Enhanced biocompatibility of $\mathrm{TiO} 2$ surfaces by highly reactive plasma J. Phys. D: Appl. Phys. 49244002

[4] Mahanti M et al 2016 The mechanism underlying calcium phosphate precipitation on titanium via ultraviolet, visable and near infrared laser-assisted biomimetic process J. Phys. D: Appl. Phys. 49304003

[5] Labay C et al 2016 Modulation of release kinetics by plasma polymerization of ampicillinloaded beta-TCP ceramics J. Phys. D: Appl. Phys. 49304004

[6] Dowling D P et al 2016 Modified drug release using atmospheric pressure plasma deposited siloxane coatings J. Phys. D: Appl. Phys. 49364005

[7] Pawlat J et al 2016 RF atmospheric plasma jet surface treatment of paper J. Phys. D: Appl. Phys. 49374001

[8] Dolci L S et al 2016 Antibody immobilization of poly(L-actic acid) nanofibers advantageously carried out by means of an non-equilibrium atmospheric plasma process J. Phys. D: Appl. Phys. 49274003

[9] Ion R et al 2016 Vertically, interconnected carbon nanowalls as biocompatible scaffolds for osteoblast cells J. Phys. D: Appl. Phys. 49274004

[10] Jofre-Reche J A et al 2016 Increased adhesion of polydimethylsiloxane (PDMS) to acrylic adhesive tape for medical use by surface treatment with an atmospheric pressure rotating plasma

jet J. Phys. D: Appl. Phys. 49334001

[11] Levchenko I et al 2016 Novel biomaterials: plasma-enabled nanostructures and functions J. Phys. D: Appl. Phys. 49273001

[12] Shelemin A et al 2016 Preparation of biomimetic nano-structured films with multi-scale roughness J. Phys. D: Appl. Phys. 49254001

[13] Bourkoula A et al 2016 Roughness threshold for cell attachment and proliferation on plasma 
micro-nanotextured polymeric surfaces J. Phys. D: Appl. Phys. 49304002

[14] Javid A et al 2016 Role of surface-electrical properties on the cell viability of carbon thing films grown in nanodomain morphology J. Phys. D: Appl. Phys. 49264001

[15] Nikiforov A 2016 Non-thermal plasma technology for the development of antimicrobial surfaces: a review J. Phys. D: Appl. Phys. 49204002

[16] Primc G et al 2016 Biodegrability of oxygen-plasma treated cellulose textile functionilized with ZnO nanoparticles as antibacterial treatment J. Phys. D: Appl. Phys. 49324002

[17] Viswan A et al 2016 Surface modification of graphite-encapsulated iron nanoparticles by RF excited $\mathrm{Ar} / \mathrm{NH} 3$ gas mixture plasma and their applications to E. coli capture J. Phys. D: Appl. Phys. 49364001

[18] Ostrikov K et al 2016 Bactericidal effects of plasma modified surface chemistry of silicon nanograss J. Phys. D: Appl. Phys. 49304001

[19] Lamont-Friedrich J S et al 2016 Chlorine-rich plasma polymer coating for the prevention of attachment of pathogenic fungal cells onto materials surfaces J. Phys. D: Appl. Phys.

49294001

[20] Yamada H et al 2016 Spectroscopy of reactive species produced by low-energy atmospheric pressure plasma on conductive target material surface J. Phys. D: Appl. Phys. 49394001

[21] Oh J S et al 2016 How to assess the plasma density delivery of RONS into tissue fluid and tissue J. Phys. D: Appl. Phys. 49304005

[22] Sasaki S et al 2016 Characterization of plasma-induced cell membrane permeabilization: focus on OH radical distribution J. Phys. D: Appl. Phys. 49334002

[23] Maheux S et al 2016 Small unilamellar liposomes as a membrane model for cell inactivation by cold atmospheric plasma treatment J. Phys. D: Appl. Phys. 49344001

J. Phys. D: Appl. Phys. 50 (2017) 040201 